\title{
Enhancing and Tuning the Response of Environmentally Sensitive Hydrogels With \\ Embedded and Interconnected Pore Networks
}

Teodora Gancheva and Nick Virgilio

Supporting Information 

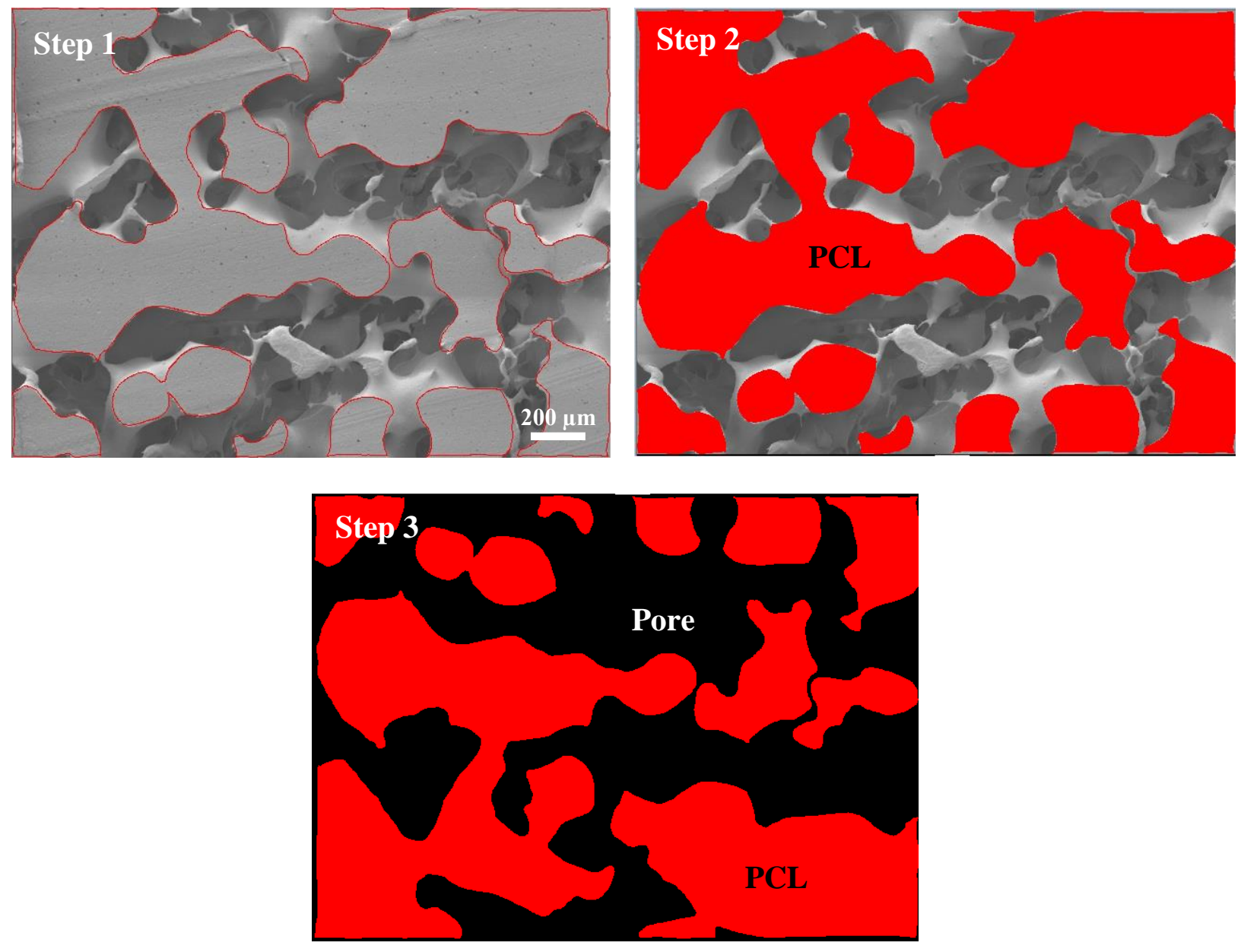

Figure S1. Successive image treatment steps for the PCL porous material obtained from the EPDM/PCL blend annealed during $240 \mathrm{~min}$.

Step 1- The PCL phase perimeter $P$ is traced using a digitizing table. The value is obtained by counting the pixels of the contour and with a proper image calibration. The specific pore surface $S$ is subsequently obtained by dividing by the micrograph's area.

Step 2- The PCL phase is subsequently filled.

Step 3- Using the ImageJ software, the micrograph is binarized. The volume fractions are calculated using the threshold function. 

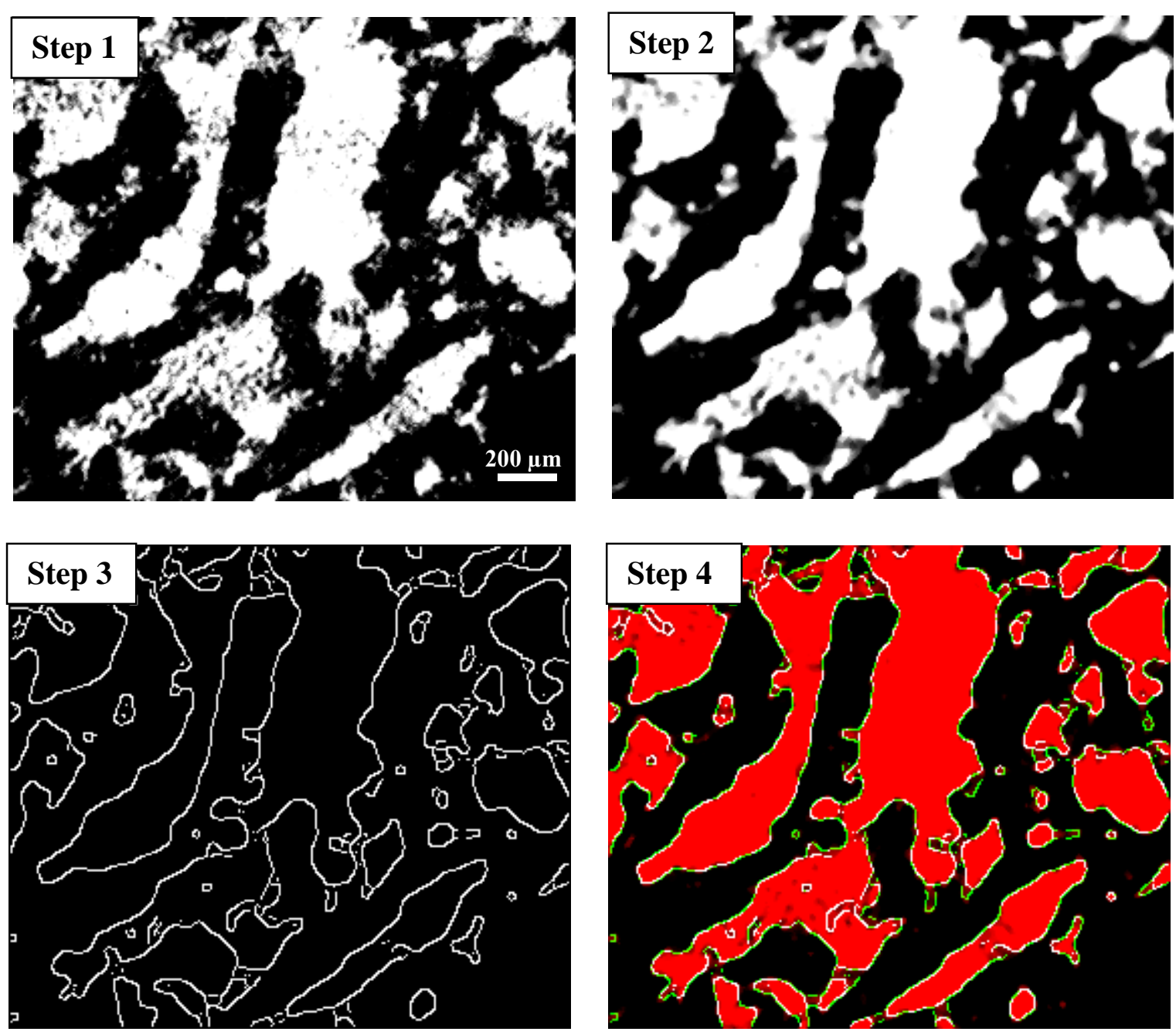

Figure S2. Successive image treatment steps for porous PNIPAam hydrogels (demonstrated for $\mathrm{G}_{240}$ ) with the ImageJ software.

Step 1- A small region from the original $\mu \mathrm{CT}$ image is selected. The brightness and contrast are adjusted to make the pores and gel phase clearer.

Step 2- The micrograph is binarized and a median filter is used to remove some noise. Then, the volume fractions are calculated using the threshold function.

Step 3- The interface perimeter $P$, with a thickness of 1 pixel, is extracted and calculated by counting the pixels in the contour, and with a proper image calibration.

Step 4- To check the superposition of the original image and the contour, colocalization is used. 

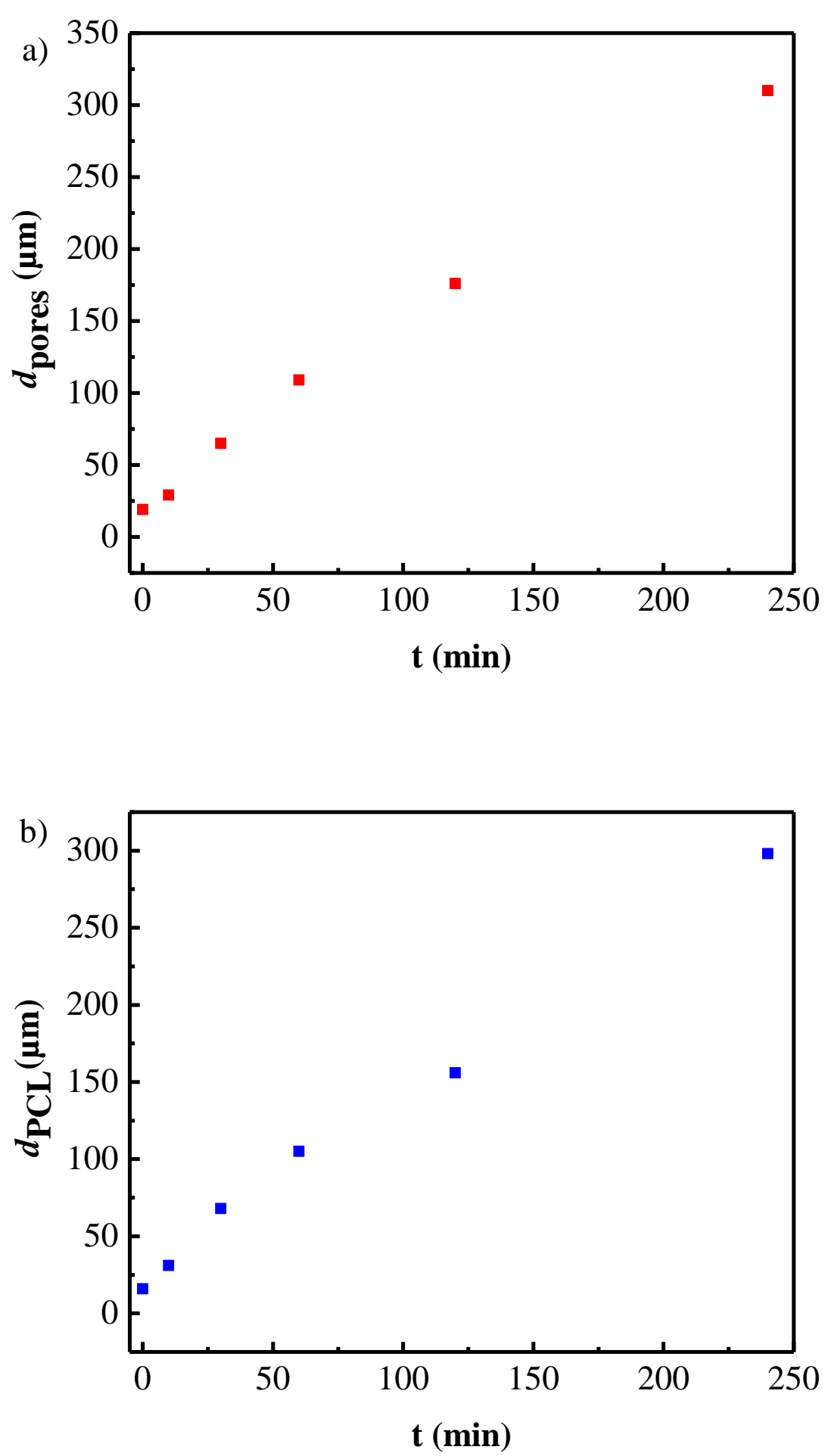

Figure S3. a) Evolution of the average pore diameter $d_{\text {pores }}$ and of b) the average PCL domain size $d_{P C L}$, as a function of quiescent annealing time, for the porous PCL molds. 

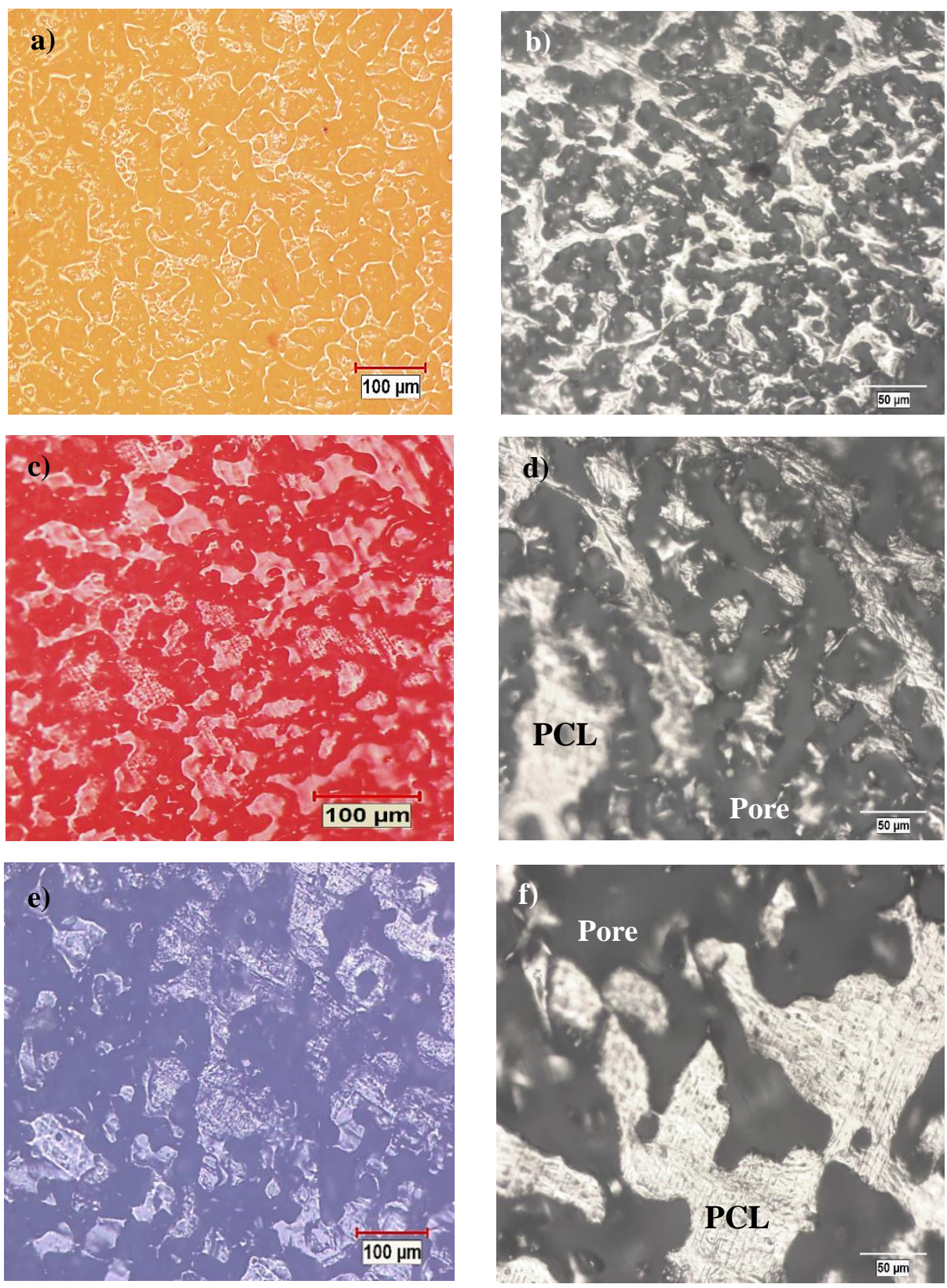

Figure S4. Optical microscopy images of porous PCL molds filled with the gelling solution, after in situ gelling (left column, gels stained with commercial food dyes (yellow, red and blue respectively), as compared to unfilled molds (right columns): a,b) 0 min (non-annealed polymer blend); c,d) porous PCL mold from blend annealed for $10 \mathrm{~min}$; e,f) $30 \mathrm{~min}$. 
a)

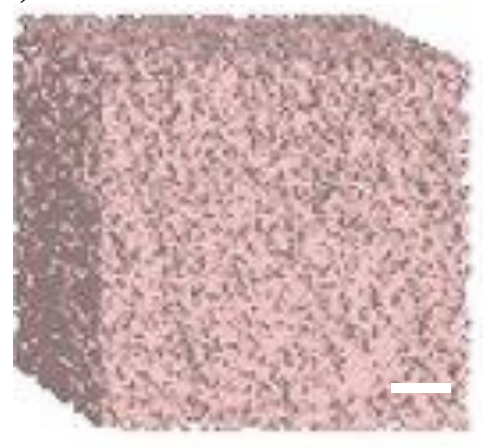

b)

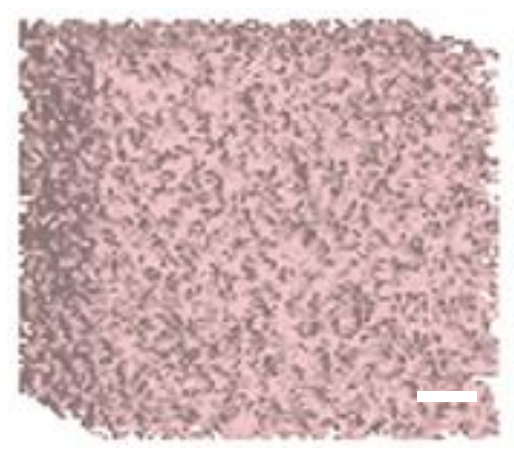

c)

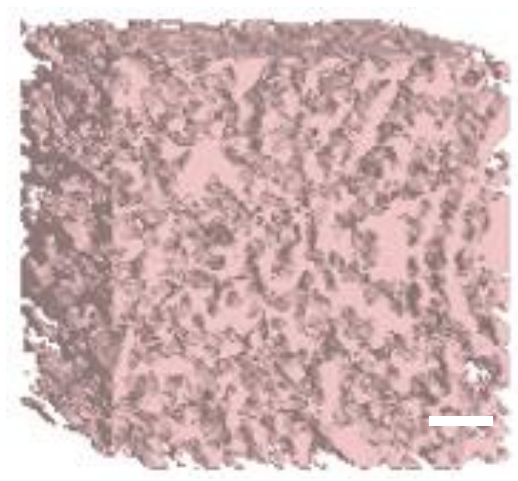

Figure S5. 3-D $\mu \mathrm{CT}$ reconstructed images of porous PNIPAam hydrogels: a) 0 min (nonannealed sample), b) $30 \mathrm{~min}$, and c) $120 \mathrm{~min}$. The scale bar corresponds to $200 \mu \mathrm{m}$. 


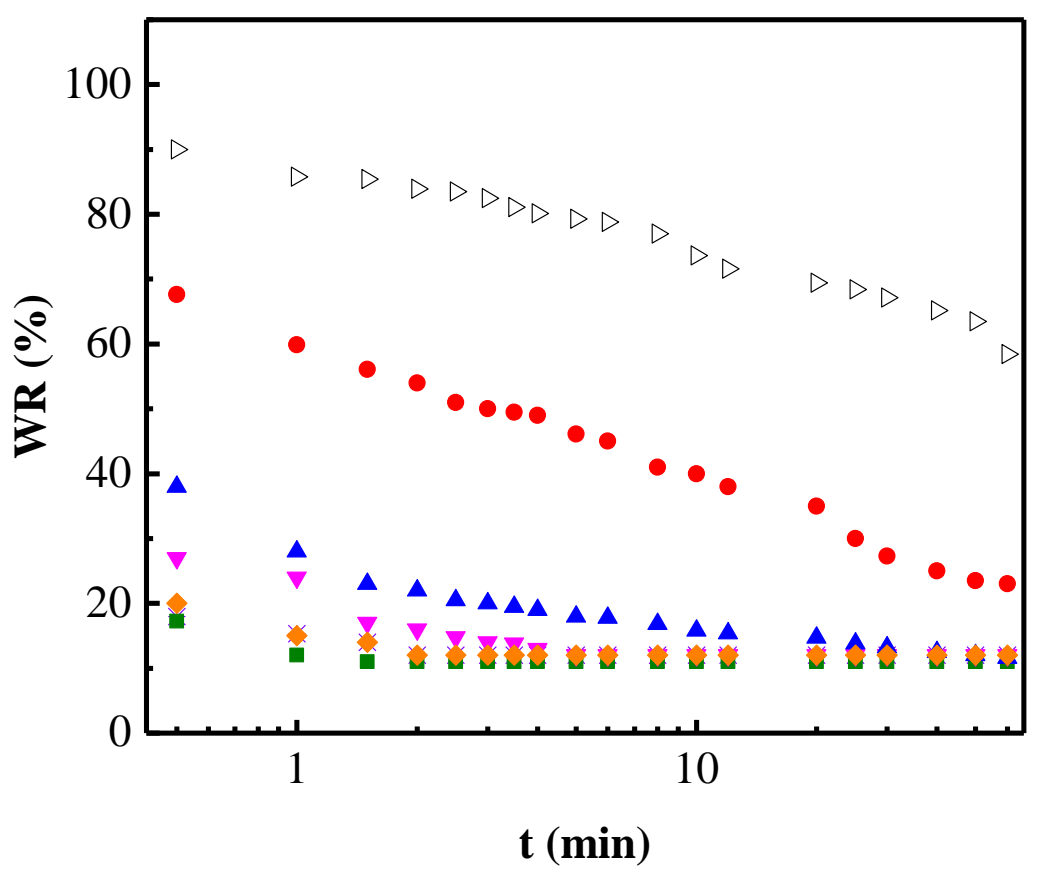

Figure S6. Water retention ratio $W R$ as a function of time for the porous PNIPAM hydrogels, when plunged in $50^{\circ} \mathrm{C}$ water, $\left(\mathrm{G}_{0} \bowtie ; \mathrm{G}_{10} * * \mathrm{G}_{30} \star ; \mathrm{G}_{60} \nabla ; \mathrm{G}_{120} \Delta ; \mathrm{G}_{240} \bullet ; \mathrm{NG} \triangleright\right)$. WR $=100 \%$ for all samples at $\mathrm{t}=0 \mathrm{~min}$. 